Trinity University

Digital Commons@ Trinity

Chemistry Faculty Research

Chemistry Department

2011

\title{
Dications of 3-Phenyl-indenylidene Dibenzo[ a.d ]cycloheptene: The Role of Charge in the Antiaromaticity of Cationic Systems
}

Nancy S. Mills

TrinityUniversity, nmills@trinity.edu

Francine E. Cheng

Trinity University

Joseph M. Baylan

Trinity University

Cornelia Tirla

Trinity University

Jennifer L. Hartmann

Trinity University

See next page for additional authors

Follow this and additional works at: https://digitalcommons.trinity.edu/chem_faculty

Part of the Chemistry Commons

\section{Repository Citation}

Mills, N. S., Cheng, F. E., Baylan, J. M., Tirla, C., Hartmann, J. L., Patel, K. C., . . McClintock, S. P. (2011). Dications of 3-phenylindenylidene dibenzo[ a. d ] cycloheptene: The role of charge in the antiaromaticity of cationic systems. Journal of Organic Chemistry, 76(2), 645-653. doi:10.1021/jo102220y 


\section{Authors}

Nancy S. Mills, Francine E. Cheng, Joseph M. Baylan, Cornelia Tirla, Jennifer L. Hartmann, Kiran C. Patel, Bart J. Dahl, and Sean P. McClintock 


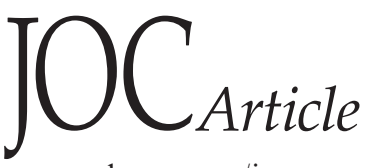

pubs.acs.org/joc

\title{
Dications of 3-Phenyl-indenylidene Dibenzo[a.d $]$ cycloheptene: The Role of Charge in the Antiaromaticity of Cationic Systems
}

\author{
Nancy S. Mills, ${ }^{*}$ Francine E. Cheng, Joseph M. Baylan, Cornelia Tirla, ${ }^{\dagger}$ \\ Jennifer L. Hartmann, Kiran C. Patel, Bart J. Dahl, ${ }^{*}$ and Sean P. McClintock \\ Department of Chemistry, Trinity University, San Antonio, Texas 78212-7200, United States. \\ $\dagger$ Current address: Department of Chemistry and Physics, University of North Carolina-Pembroke, \\ North Carolina. ${ }^{*}$ Current address: Department of Chemistry, University of Wisconsin-Eau Claire, \\ Eau Claire, Wisconsin. \\ nmills@trinity.edu
}

Received November 6, 2010

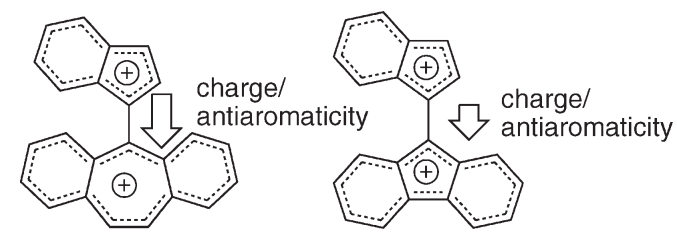

Dications of 9-(3-phenyl-1H-inden-1-ylidene)-5H-dibenzo[a,d]cycloheptene, $\mathbf{5}^{\mathbf{2 +}}$, were prepared by oxidation with $\mathrm{SbF}_{5}$ in $\mathrm{SO}_{2} \mathrm{ClF}$, and their magnetic behavior was compared to dications of 9-(3-phenyl-1H-inden-1-ylidene)-9H-fluorene, $\mathbf{2}^{\mathbf{2}+}$. The good correlation between the experimental ${ }^{1} \mathrm{H}$ NMR shifts for the dications that were oxidized cleanly and the chemical shifts calculated by the GAIO method supported the use of the nucleus independent chemical shifts, NICS, to evaluate the antiaromaticity of the indenyl systems of $\mathbf{2}^{\mathbf{2 +}} / \mathbf{5}^{\mathbf{2 +}}$ and their unsubstituted parent compounds, $\mathbf{6}^{\mathbf{2 +}}$ and $\mathbf{7}^{\mathbf{2 +}}$, as well as the antiaromaticity of the fluorenyl system of $\mathbf{2}^{\mathbf{2 +}} / \mathbf{7}^{\mathbf{2 +}}$ and the aromaticity of the dibenzotropylium system of $5^{2+} / 6^{2+}$. Antiaromaticity was shown to be directly related to the amount of charge in the antiaromatic systems, with the antiaromatic systems more responsive to changes in the calculated NBO charge than the aromatic systems. The antiaromaticity was also shown to be directly related to the amount of delocalization in the ring system. The aromaticity of the dibenzotropylium system was much less responsive to changes in the amount of charge in the tropylium system, because the aromatic system was much more completely delocalized. Thus, antiaromatic species are more sensitive probes of delocalization than aromatic ones.

\section{Introduction}

Substantial interest exists in the properties of antiaromatic species ${ }^{1-6}$ but there are few examples of antiaromatic species that can be prepared and characterized experimentally. We have successfully prepared a number of hydrocarbon dications

(1) Tidwell, T. Chem. Rev. 2001, 101, 1333-1348

(2) Breslow, R. In Magnetic Properties of Organic Materials; Lahti, P. M., Ed.; Marcel Dekker: New York, 1999; pp 27-40.

(3) Wiberg, K. B. Chem. Rev. 2001, 101, 1317-1331.

(4) Bally, T. Angew. Chem., Int. Ed. 2006, 45, 6616-6619.

(5) Herges, R. Nature 2007, 450, 36-37.

(6) Breslow, R.; Foss, F. W., Jr. J. Phys.: Cond. Matt. 2008, 20, 374104/ 374101-374104/374107.

(7) Piekarski, A. M.; Mills, N. S.; Yousef, A. J. Am. Chem. Soc. 2008, 130, 14883-14890.

DOI: $10.1021 /$ jo $102220 y$

Published on Web 12/29/2010 that contain an antiaromatic cationic fluorenyl system, ${ }^{7-18}$ for example, $\mathbf{1}^{\mathbf{2}}$, and have recently extended those studies to

\footnotetext{
(8) Dahl, B. J.; Mills, N. S. J. Am. Chem. Soc. 2008, 130, 10179-10186.

(9) Dahl, B. J.; Mills, N. S. Org. Lett. 2008, 10, 5605-5608.

(10) Herndon, W. C.; Mills, N. S. J. Org. Chem. 2005, 70, 8492-8496

(11) Mills, N. S.; Tirla, C.; Benish, M. A.; Rakowitz, A. J.; Bebell, L. M.; Hurd, C. M. M.; Bria, A. L. M. J. Org. Chem. 2005, 70, 10709-10716.

(12) Mills, N. S.; Benish, M. M.; Ybarra, C. J. Org. Chem. 2002, 67, $2003-$ 2012.

(13) Mills, N. S. J. Org. Chem. 2002, 67, 7029-7036.

(14) Levy, A.; Rakowitz, A.; Mills, N. S. J. Org. Chem. 2003, 68, 39903998.

(15) Mills, N. S.; Levy, A.; Plummer, B. F. J. Org. Chem. 2004, 69, 66236633.

(16) Mills, N. S.; Malinky, T.; Malandra, J. L.; Burns, E. E.; Crossno, P. J. Org. Chem. 1999, 64, 511-517.
}

(17) Mills, N. S. J. Am. Chem. Soc. 1999, 121, 11690-11696.

J. Org. Chem. 2011, 76, 645-653

645 
dications containing an antiaromatic indenyl cation, ${ }^{19} \mathbf{2}^{\mathbf{2 +}}$, and to dianions containing an antiaromatic dibenzocycloheptenyl system, ${ }^{7} \mathbf{3}^{\mathbf{2}}$. These systems have allowed us to confirm that the aromaticity/antiaromaticity suggested by nucleus independent chemical shifts is inversely related to the square of the area of the ring system, ${ }^{20}$ have allowed an assessment of the relationship between stability and magnetic measures of antiaromaticity, ${ }^{11}$ and have demonstrated that the lack of bond length alternation seen as a characteristic of aromatic compounds is also present in antiaromatic species. ${ }^{7}$

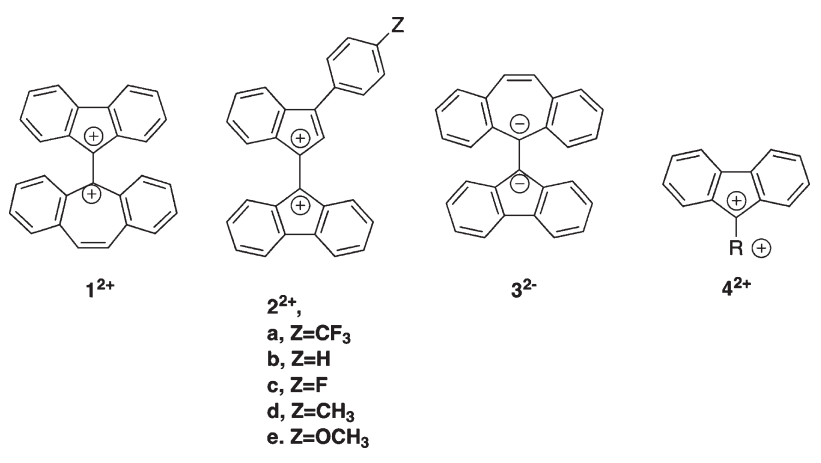

The dicationic species that we have previously prepared have all contained an antiaromatic ring system that is connected to a substituent, $\mathbf{4}^{\mathbf{2}+}$, that can be a cyclic system as well as an acylic substituent. The cyclic substituent can be an aromatic ion, as in $\mathbf{1}^{\mathbf{2 +}}$, or an antiaromatic ring system, as in $\mathbf{2}^{\mathbf{2}+}$. The antiaromaticity of the fluorenyl or indenyl ring system is affected by the substituent, which is difficult to rationalize because the fluorenyl ring is not planar with its substituent $\mathrm{R}^{+}$. That is, the calculated geometry of $\mathbf{1}^{\mathbf{2}+}$ shows that the fluorenyl system is perpendicular to the dibenzotropylium system, ${ }^{13}$ while for species such as $\mathbf{2}^{\mathbf{2}+}$, the calculated geometry shows that the dihedral between the indenyl and fluorenyl systems is $\sim 50^{\circ}$, vide infra. If the ring systems are not able to interact through resonance, what is the mode of interaction?

We have chosen to examine one aspect of this through the preparation of indenyl cations with 3-phenyl substituents that are connected to a dibenzotropylium system, $\mathbf{5}^{\mathbf{2}}$. By changing the nature of the $p$-substituent on the phenyl ring, we can moderate the electronic environment in the indenyl system and examine the effects of that interaction on the antiaromaticity of the indenyl system as well as on the aromatic dibenzotropylium system (described as the tropylium system in the rest of the paper). We have previously reported the preparation and characterization of derivatives of $\mathbf{2}^{\mathbf{2}+}$ and can use those results to understand the effect of changes in the indenyl system on its antiaromaticity as well as on the antiaromatic fluorenyl system. The comparison of $\mathbf{2}^{\mathbf{2 +}}$ with $\mathbf{5}^{\mathbf{2}+}$ will also help to understand the effect of the

(18) Malandra, J. L.; Mills, N. S.; Kadlecek, D. E.; Lowery, J. A. J. Am. Chem. Soc. 1994, 116, 11622-11624.

(19) Mills, N. S.; Llagostero, K. B.; Tirla, C.; Gordon, S.; Carpenetti, D. J. Org. Chem. 2006, 71, 7940-7946.

(20) Mills, N. S.; Llagostera, K. B. J. Org. Chem. 2007, 72, 9163-9169. fluorenyl/tropylium system on the antiaromaticity of the indenyl system.

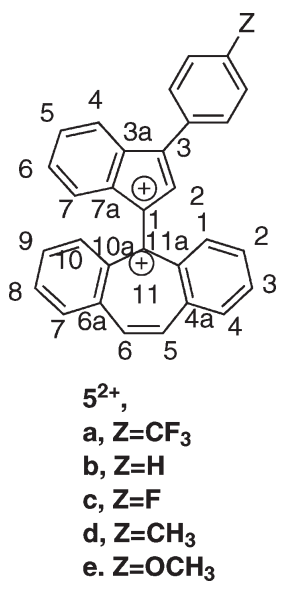

Traditionally, the characterization of antiaromatic species has been through theoretical calculation, ${ }^{3,21,22}$ which opens the conclusions to concerns about how the calculation were done, including issues of basis set choice. When theoretical data can be shown to have a good correlation with experimental data, the theoretical data is viewed as more reliable, particularly by experimental chemists. We have been able to successfully use this approach to "validate" measures of aromaticity like nucleus independent chemical shifts, NICS, which have no direct experimental analogue. NICS are the chemical shifts calculated for a ghost atom placed $1 \AA$ above the planar ring system. ${ }^{23}$ The chemical shift is a tensor, and the component of the chemical shift parallel to the $\pi$-system has been shown to most reliably correlate with other measures of aromaticity, so we will draw our conclusions from these NICS values, designated as NICS $(1)_{z z} \cdot{ }^{24}$ If a good correlation exists between the experimental shifts and the NMR chemical shifts calculated by the same method as the NICS values, the method for calculation of those NMR shifts, and by extension the NICS values, are considered to be more reliable. In addition, a good correlation between experimental and calculated shifts also supports the quality of the calculated geometry for these reactive species.

Our approach with this work will be to examine the effect on the antiaromaticity of the indenyl systems of changing the substituent $\mathbf{Z}$ on $\mathbf{2}^{\mathbf{2 +}}$ and $\mathbf{5}^{\mathbf{2}+}$, to see if the changes are transmitted to the fluorenyl and tropylium systems, and if so, to explore any differences in the antiaromaticity/aromaticity of the latter two systems. We will use the NICS values, rather than the ${ }^{1} \mathrm{H}$ NMR shifts to assess the antiaromaticity of the indenyl/fluorenyl systems, or the aromaticity of the tropylium system. There is sufficient variability in the ${ }^{1} \mathrm{H}$ NMR shifts that they can only be used to evaluate aromaticity in a quantitative sense for compounds that are carefully

(21) Schleyer, P. v. R.; Freeman, P. K.; Jiao, H.; Goldfuss, B. Angew. Chem., Int. Ed. Engl. 1995, 34, 337-340.

(22) Fowler, P. W.; Zansai, R.; Cadioli, B.; Steiner, E. Chem. Phys. Lett. 1996, 251, 132-140.

(23) Schleyer, P. v. R.; Maerker, C.; Dransfeld, A.; Jiao, H.; Hommes, N. J. v. E. J. Am. Chem. Soc. 1996, 118, 6317-6318.

(24) Fallah-Bagher-Shaidaei, H.; Wannere, C. S.; Corminboeuf, C.; Puchta, R.; Schleyer, P. v. R. Org. Lett. 2006, 8, 863-866. 


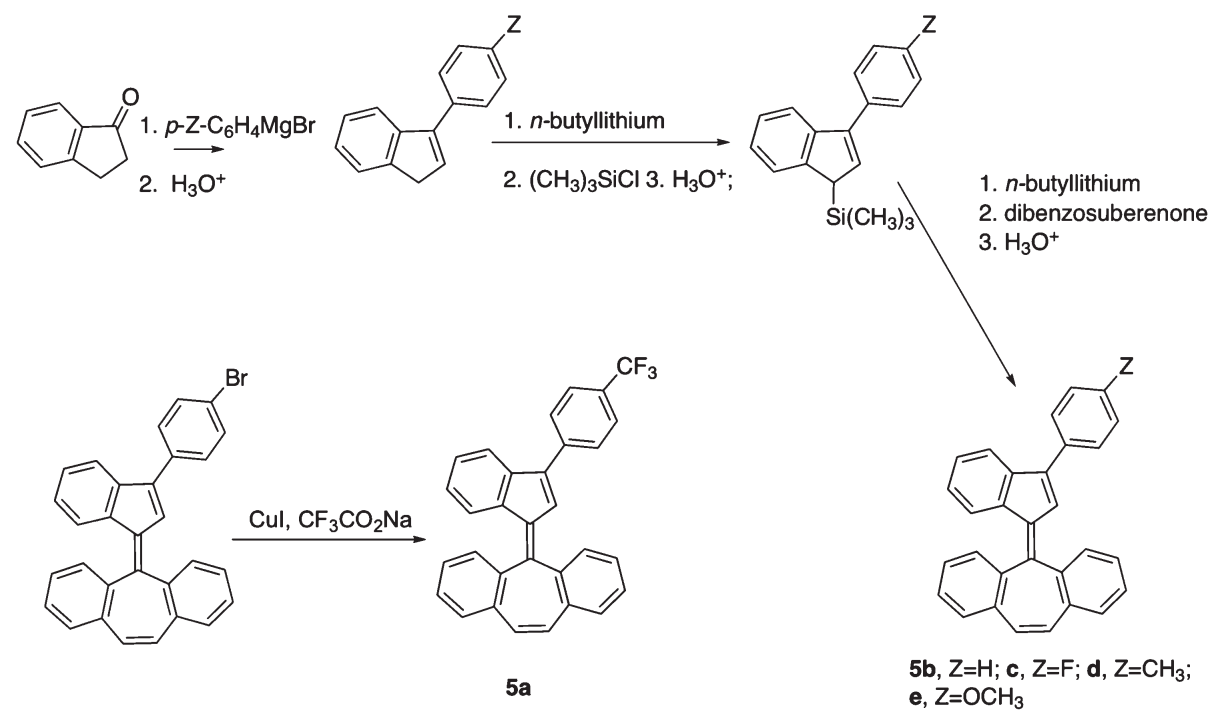

matched in terms of structure. ${ }^{25}$ We have chosen to evaluate the effect of the substituent $\mathrm{Z}$ through changes in the distribution of charge of the indenyl, fluorenyl, and tropylium systems. In theory, charge on a carbon should be able to be probed experimentally but there are issues with this approach. ${ }^{26}$ As well there are issues with the calculation of charge. ${ }^{27}$ Recently, Baldridge, et al., ${ }^{28}$ have observed a good relationship between experimentally determined charges and those calculated by natural population analysis, natural bond order (NBO) calculations. ${ }^{27}$ We will use the NBO calculated charges to observe changes in charge distribution and will couple those with the NICS values.

There is one caveat with this study. We are making antiaromatic species and there is no guarantee that the species we seek will be stable enough for characterization. We were able only to prepare $\mathbf{2 a}-\mathbf{c}^{\mathbf{2}+}$ with high enough purity to have confidence in the assignment of their ${ }^{1} \mathrm{H}$ NMR chemical shifts. We report here the preparation and characterization of $\mathbf{5 b}-\mathbf{d}^{\mathbf{2}+}$ by oxidation of the unsaturated precursor and were unable to get sufficiently high quality data for the oxidation of $\mathbf{5 a}$ and $\mathbf{5} \mathbf{e}^{\mathbf{2}+}$ to make reliable assignments. We argue, however, that if the calculated and experimental shifts for $\mathbf{5 b}-\mathbf{d}^{\mathbf{2}+}$ show a linear relationship, there is no reason to assume that the spectra of $\mathbf{5} \mathbf{a}$ and $\mathbf{5 e}$ would not also show the same linear relationship. Thus we can interpolate the data with calculated data and be in a better position to understand trends in the effects of substituent Z.

\section{Results and Discussion}

Preparation of Substituted 3-Phenyl-indenylidene Dibenzocycloheptenes and their Oxidation to Dications $\mathbf{5}^{\mathbf{2}}$. The synthetic strategy for preparation of the indenylidene diben-

(25) Mitchell, R. H. Chem. Rev. 2001, 101, 1301-1316.

(26) Wiberg, K. B.; Hammer, J. D.; Keith, T. A.; Zilm, K. Tetrahedron Lett. 1997, 38, 323-326.

(27) Reed, A. E.; Weinstock, R. B.; Weinhold, F. J. Chem. Phys. 1985, 83, 735-746.

(28) Yerushalmi, R.; Scherz, A.; Baldridge, K. K. J. Am. Chem. Soc. 2004, $126,5897-9505$. zocycloheptenes is shown in Scheme 1. Reaction of 1-indanone with an appropriately substituted phenyl Grignard reagent, followed by dehydration gave 1-( $p$-substituted-phenyl)indene. Deprotonation of the indene with $n$-butyllithium followed by reaction with trimethylsilyl chloride gave the TMS indene derivative. Subsequent deprotonation with $n$-butyllithium was followed by reaction with dibenzosuberenone, 5H-dibenzo[a,d]cyclohepten-5-one, to give the indenylidene dibenzo[a.d]cycloheptenes via Peterson olefination. The trifluoromethyl derivative, 2a, was synthesized from the $p$-bromophenyl derivative by reaction with sodium trifluoroacetate with copper(I) iodide.

Preparation of $\mathbf{5}^{\mathbf{2}}$. NMR Shifts. Oxidation of $\mathbf{5 b}-\mathbf{5 d}$ with $\mathrm{SbF}_{5}$ in $\mathrm{SO}_{2} \mathrm{ClF}$ gave $\mathbf{5 b}^{\mathbf{2 +}} \mathbf{- 5} \mathbf{d}^{\mathbf{2}+}$. The ${ }^{1} \mathrm{H}$ NMR spectrum of $\mathbf{5} \mathbf{b}^{\mathbf{2 +}}$ is shown in Figure 1; spectra of $\mathbf{5} \mathbf{c}^{\mathbf{2 +}}$ and $\mathbf{5} \mathbf{d}^{\mathbf{2}+}$ can be found in the Supporting Information. Assignments were made via 2D COSY spectra and comparison with spectra calculated using the GIAO method with basis set B3LYP/ 6-311+g(d,p) on structures optimized at the B3LYP level. The experimental spectrum of $\mathbf{5} \mathbf{b}^{\mathbf{2 +}}$ is not as pure as that of $\mathbf{5 d}^{\mathbf{2 +}}$. There are additional peaks between 6.3 and $6.75 \mathrm{ppm}$, an increased integration in the region between 6.9 and 7.8 and a very large peak around 8 , which may also contain protonated water. However the resolution is much better than seen in the spectrum of $\mathbf{5} \mathbf{d}^{\mathbf{2}}$. The presence of the methyl substituent in $\mathbf{5 d}^{\mathbf{2}+}$ would be expected to provide additional stabilization to the dication. Oxidation of $\mathbf{5} \mathbf{c}$ resulted in the formation of the dication, but the spectrum was much less clean. It was possible to make assignments for $\mathbf{5} \mathbf{c}^{\mathbf{2}+}$ based primarily on the COSY spectrum and the calculated chemical shifts. The experimental and calculated spectra are given in Table 1.

The calculated shifts clearly show the upfield shift anticipated for the antiaromatic indenyl system and the down-field shift for the aromatic dibenzotropylium system. These same regions are apparent in the experimental spectrum. A plot of calculated vs experimental shifts for $\mathbf{5} \mathbf{b}^{\mathbf{2}+}-\mathbf{5} \mathbf{d}^{\mathbf{2}+}$ is shown in Figure 2 and shows excellent agreement, with $R=0.987$. The quality of the agreement between experimental and calculated shifts gives support to NICS calculations using the same method and basis sets, vide infra. Attempts to oxidize 


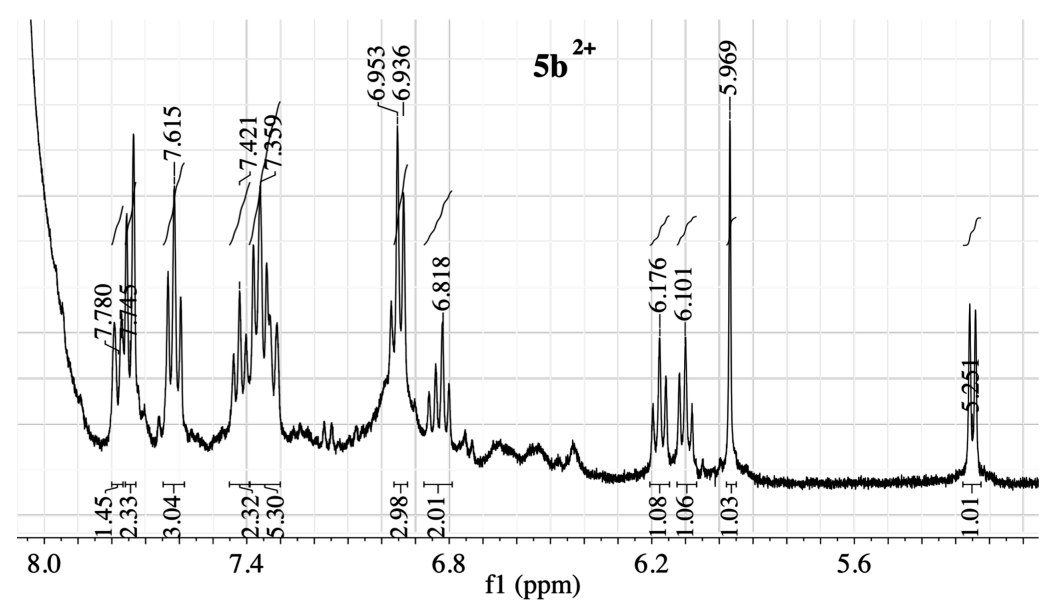

FIGURE 1. ${ }^{1} \mathrm{H}$ NMR spectrum of $\mathbf{5 b}^{\mathbf{2}+}$ in $\mathrm{SO}_{2} \mathrm{ClF}$, with $d_{6}$ - acetone/TMS as an external reference.

TABLE 1. Calculated ${ }^{a}{ }^{1} \mathrm{H}$ NMR Shifts for $5 \mathrm{a}-\mathrm{e}^{2+}$ and Experimental ${ }^{b}$ Shifts for $5 b-d^{2+}$

\begin{tabular}{|c|c|c|c|c|c|c|c|c|}
\hline & $5 a^{2+}$ & $5 b^{2+}$ & $5 b^{2+}$ & $5 c^{2+}$ & $5 c^{2+}$ & $5 d^{2+}$ & $5 d^{2+}$ & $5 \mathrm{e}^{2+}$ \\
\hline $\begin{array}{l}\text { proton } \\
\text { number }\end{array}$ & calcd & calcd & $\exp$ & calcd & $\exp$ & calcd & $\exp$ & calcd \\
\hline \multicolumn{9}{|c|}{ Indenyl system } \\
\hline 2 & 6.399 & 6.740 & 5.969 & 5.753 & 5.700 & 6.827 & 5.817 & 6.844 \\
\hline 4 & 7.576 & 6.429 & 5.258 & 6.915 & 6.692 & 7.991 & 6.458 & 8.084 \\
\hline 5 & 6.984 & 7.324 & 6.099 & 6.679 & 5.959 & 7.431 & 6.024 & 7.295 \\
\hline 6 & 7.028 & 7.382 & 6.179 & 6.673 & 5.880 & 7.365 & 5.935 & 7.243 \\
\hline 7 & 6.271 & 7.910 & 6.818 & 5.461 & 5.056 & 6.473 & 5.090 & 6.514 \\
\hline \multicolumn{9}{|c|}{ Dibenzotropylium system } \\
\hline 1,10 & 10.017 & 9.692 & $\sim 8.0$ & 8.138 & 7.673 & 9.609 & $\sim 8.0$ & 9.553 \\
\hline 2,9 & 8.629 & 8.797 & 7.359 & 7.621 & 7.095 & 8.761 & 7.089 & 8.477 \\
\hline 3,8 & 8.880 & 9.063 & 7.656 & 8.072 & 7.494 & 9.041 & 7.373 & 8.833 \\
\hline 4,7 & 8.925 & 9.134 & 7.737 & 7.888 & 7.356 & 9.119 & 7.512 & 8.892 \\
\hline 5,6 & 9.289 & 9.518 & $\sim 8.0$ & 8.314 & 8.097 & 9.507 & $\sim 8.0$ & 9.259 \\
\hline \multicolumn{9}{|c|}{ Phenyl ring } \\
\hline o & 8.380 & 8.376 & $\sim 6.95$ & 7.043 & 7.206 & 8.302 & 7.029 & 8.050 \\
\hline o & 8.711 & 8.746 & 7.341 & 7.694 & 7.727 & 8.714 & 7.583 & 9.094 \\
\hline $\mathrm{m}$ & 8.030 & 8.113 & $\sim 6.95$ & 6.805 & 6.247 & 7.926 & 6.607 & 7.271 \\
\hline $\mathrm{m}$ & 8.190 & 8.264 & $\sim 6.95$ & 6.962 & 6.384 & 8.106 & 6.825 & 7.436 \\
\hline $\mathrm{p}$ & & 8.757 & 7.419 & & & & & \\
\hline subst & & & & & & 2.671 & 1.511 & 4.274 \\
\hline
\end{tabular}

${ }^{a}$ Shifts calculated with the GIAO method with the inclusion of solvent using the PCM method using DMSO as the solvent with basis set B3LYP/6-311 $+\mathrm{g}(\mathrm{d}, \mathrm{p})$ on geometries optimized at the B3LYP/6-31 g(d) level. ${ }^{b}$ Chemical shifts determined in $\mathrm{SO}_{2} \mathrm{ClF}$, referenced to an external capillary with $d_{6}$-acetone and TMS.

$\mathbf{5} \mathbf{a}^{\mathbf{2 +}}$ and $\mathbf{5} \mathbf{e}^{\mathbf{2 +}}$ were unsuccessful. It is not surprising that the highly electron-withdrawing trifluoromethyl substituent would destabilize the carbocation, but we had anticipated that the methoxy substituent on $\mathbf{5 e}^{\mathbf{2 +}}$ would stabilize the carbocation. However, we have seen complexation of $\mathrm{SbF}_{5}$ with a methoxy substituent in other systems ${ }^{16}$ and its complexation would reduce the stabilizing effect of the methoxy group.

Nucleus Independent Chemical Shifts. The ring current model of aromaticity predicts a magnetic environment in the center of an aromatic ring that is opposite in sign to that of the periphery, resulting in protons that are shifted downfield when on the periphery of the aromatic species and would be shifted upfield if present in the center of the aromatic ring. These effects would be opposite for an antiaromatic species

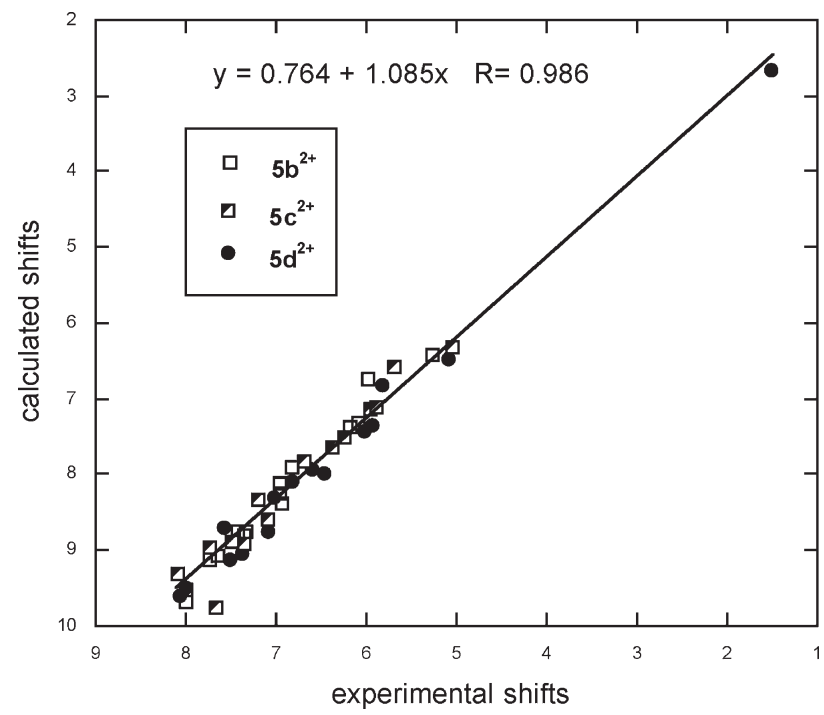

FIGURE 2. Comparison of calculated and experimental shifts for $\mathbf{5} \mathbf{b}^{\mathbf{2}}, \mathbf{5} \mathbf{c}^{\mathbf{2 +}}$, and $\mathbf{5 d}^{\mathbf{2}+}$. See Table 1 for details of the calculations and experimental conditions.

and these predictions have been given experimental support in the ${ }^{1} \mathrm{H}$ NMR characterization of [18]annulene and its dianion. ${ }^{29}$ Nucleus independent chemical shifts, NICS, are based on this phenomenon, with the calculation of the magnetic shielding tensor for a dummy atom placed in the center of the ring system. ${ }^{23}$ NICS for an aromatic system have a negative sign; those for an antiaromatic system have a positive sign. As mentioned earlier, the method has been refined by the placement of the dummy atom $1 \AA$ above the plane of the ring to avoid effects of electrons in $\sigma$-bonds in small ring systems, and by the use of the component of the magnetic shielding tensor that is perpendicular to the plane of the ring, NICS $(1)_{z z} \cdot{ }^{24}$ We have recently shown that the summation of the NICS values for each ring in a polycyclic system is an accurate measure of the aromaticity/antiaromaticity of the polycyclic system. We also determined that the magnitude of

(29) Oth, J. F. M.; Woo, E. P.; Sondheimer, F. J. Am. Chem. Soc. 1973, $95,7337-7345$.

648 J. Org. Chem. Vol. 76, No. 2, 2011 
TABLE 2. Nucleus Independent Chemical Shifts ${ }^{a}$, NICS, for $5 \mathrm{a}-\mathrm{e}^{2+}$ and $6^{2+}$

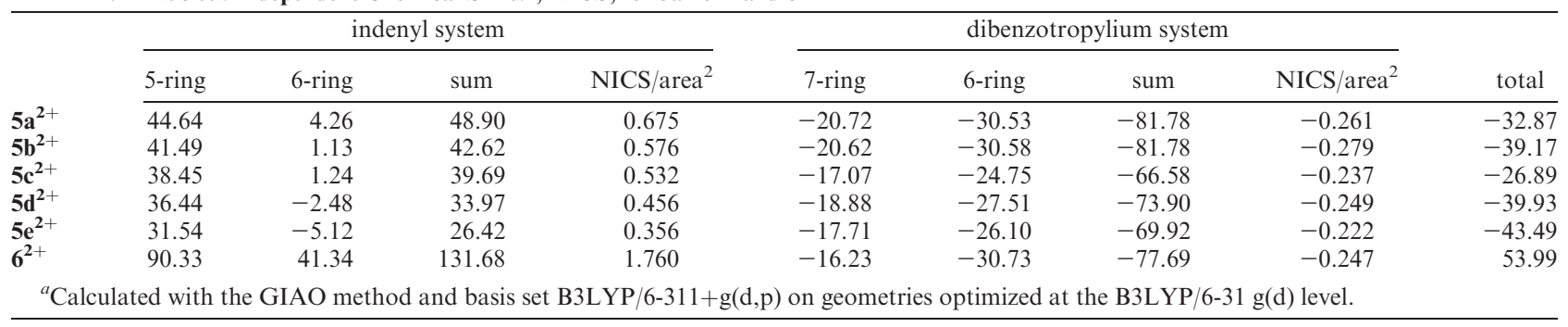

a)

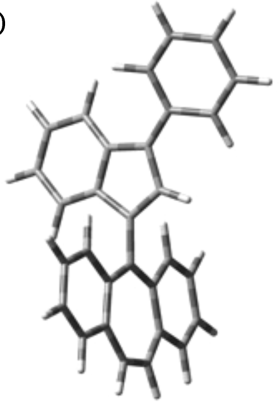

b)

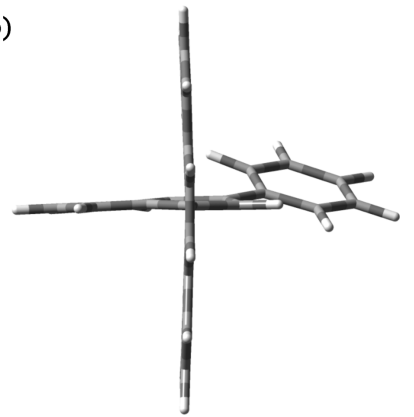

FIGURE 3. Optimized geometry for $\mathbf{5 b}^{\mathbf{2}+}$. (a) View showing all atoms. (b) Orientation showing the perpendicular relationship of the indenyl and tropylium ring systems.

the NICS value is inversely related to the square of the ring area, which must be considered in the use of NICS to evaluate aromaticity/antiaromaticity in ring systems of different sizes. ${ }^{20}$

The calculated NICS $(1)_{z z}$ values for $\mathbf{5} \mathbf{a}-\mathbf{e}^{\mathbf{2}+}$ are given in Table 2, along with the NICS $(1)_{z z}$ values of the unsubstituted indenylidene dibenzotropylium dication, $\mathbf{6}^{\mathbf{2}+}$, vide infra. The antiaromaticity of the indenyl systems are evident through the positive sign of NICS and the aromaticity of the dibenzotropylium systems is supported by their negative NICS. They were calculated using the GIAO method with basis set B3LYP $/ 6-311+\mathrm{g}(\mathrm{d}, \mathrm{p})$ on geometries optimized at the B3LYP/6-31 g(d) level using Gaussian03. As noted previously, this approach gives good agreement between calculated and experimental ${ }^{1} \mathrm{H}$ NMR shifts. It is clear that the more electron-deficient the 3-phenyl substituent, the more antiaromatic the indenyl system becomes and that the greatest antiaromaticity in the indenyl system is seen for $\mathbf{6}^{\mathbf{2 +}}$, which lacks the stabilization of the phenyl ring. The optimized geometry of $\mathbf{5 a}-\mathbf{e}^{\mathbf{2}+}$ reveals that the indenyl and dibenzotropylium ring systems are perpendicular to each other, as is shown in Figure 3 for $\mathbf{5 b}^{\mathbf{2 +}}$.

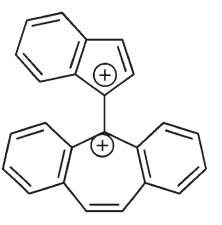

$6^{2+}$

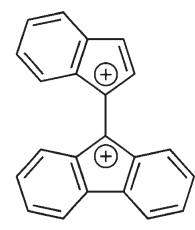

$7^{2+}$

Comparison with Related Dications: NICS. As was described previously, we were interested in the effect on the antiaromaticity of the indenyl system of replacing the aromatic

dibenzotropylium system with a fluorenyl cation, that is, comparing the antiaromaticity of the indenyl system of $\mathbf{5}^{\mathbf{2}}$ with that of $\mathbf{2}^{\mathbf{2}+}$, and on the effect of those changes on the tropylium and fluorenyl systems, respectively. We had previously prepared dications $\mathbf{2 a}-\mathbf{c}^{\mathbf{2 +}}$ by oxidation with $\mathrm{SbF}_{5}{ }^{19}$ To assess the effect on antiaromaticity/aromaticity of replacing the dibenzotropylium system with a fluorenyl system, we rely on comparison of NICS. However, the NICS $(1)_{z z}$ values for $\mathbf{2 a}-\mathbf{e}^{\mathbf{2 +}}$ previously reported were done at a different basis set and used the average of the three magnetic tensors rather than the tensor perpendicular to the plane of the ring system, so the values were recalculated and are reported in Table 3, along with that of the unsubstituted indenylidene fluorene dication, $\mathbf{7}^{\mathbf{2}}$. The comparison of experimental vs ${ }^{1} \mathrm{H}$ NMR shifts for $\mathbf{2} \mathbf{a}-\mathbf{c}^{\mathbf{2}+}$ is given in the Supporting Information. The agreement is very good, $R=$ 0.967 , and improves to 0.984 when data for $\mathbf{5 b}-\mathbf{d}^{\mathbf{2}+}$ is included. The excellent agreement when both systems are examined supports the use of calculations with GIAO at the B3LYP $/ 6-311+g(d, p)$ level for NICS.

As was true for $\mathbf{5}^{\mathbf{2}}$, the antiaromaticity of the indenyl system decreased with phenyl substitution and as $Z$ became more electron-rich. The antiaromaticity of the fluorenyl system behaved in an analogous way. We asked what role the substituted phenyl ring could be playing in affecting the antiaromaticity of the indenyl and fluorenyl ring systems and it seemed reasonable that the effect of $Z$ might be on the stabilization of the positive charge in the indenyl system; that as $\mathrm{Z}$ became more electron-donating, it would stabilize the positive charge by delocalizing it into the phenyl ring. We chose to calculate the charges by the natural population analysis method, giving natural bond order (NBO) charges which have been shown to represent experimental charges reliably. ${ }^{28}$ The table of the calculated NBO charges for each atom of $\mathbf{2}^{\mathbf{2 +}}, \mathbf{5}^{\mathbf{2 +}}, \mathbf{6}^{\mathbf{2 +}}$ and $\mathbf{7}^{\mathbf{2 +}}$ can be found in the Supporting Information. Table 4 contains the sum of the charges for each ring system of $\mathbf{2}^{\mathbf{2 +}}, \mathbf{5}^{\mathbf{2}+}, \mathbf{6}^{\mathbf{2 +}}$ and $\mathbf{7}^{\mathbf{2}+}$

Examining the unsubstituted systems, $\mathbf{6}^{\mathbf{2}+}$ and $\mathbf{7}^{\mathbf{2}}$ first, the charges are unevenly partitioned between the ring systems, with slightly more positive charge on the fluorenyl and tropylium systems. This may simply reflect the greater ability of the larger system to support a positive charge. As would be expected, the presence of the phenyl substituent in the 3 -position causes additional delocalization of the positive charge throughout the entire indenyl system, with greater movement of charge into that system as $\mathrm{Z}$ becomes more electron-donating and therefore more capable of stabilizing the charge. While the magnitude of the sum of the charges on the phenyl substituent as a function of substituent are not equal, for example, the charge on the anisyl ring of $\mathbf{2} \mathbf{e}^{\mathbf{2 +}}$ is 
TABLE 3. Nucleus Independent Chemical Shifts, ${ }^{a} \operatorname{NICS}(1)_{z z}$, for $2 \mathrm{a}-\mathrm{e}^{2+}$ and $7^{2+}$

\begin{tabular}{|c|c|c|c|c|c|c|c|c|c|}
\hline & \multicolumn{4}{|c|}{ indenyl system } & \multicolumn{4}{|c|}{ fluorenyl system } & \multirow[b]{2}{*}{ total } \\
\hline & 5-ring & 6-ring & sum & NICS/area ${ }^{2}$ & 5-ring & 6-ring & sum & NICS/area ${ }^{2}$ & \\
\hline $2 a^{2+}$ & 43.89 & 7.03 & 50.91 & 0.677 & 68.61 & 25.93 & 120.48 & 0.631 & 171.39 \\
\hline $2 \mathbf{b}^{2+}$ & 40.38 & 4.28 & 44.66 & 0.596 & 66.99 & 24.30 & 115.60 & 0.605 & 160.25 \\
\hline $2 c^{2+}$ & 38.15 & 2.40 & 40.55 & 0.539 & 66.91 & 23.97 & 114.78 & 0.601 & 155.41 \\
\hline $2 d^{2+}$ & 33.22 & -2.47 & 30.75 & 0.410 & 65.70 & 22.57 & 110.83 & 0.579 & 141.57 \\
\hline $2 \mathrm{e}^{2+}$ & 28.22 & -5.10 & 23.11 & 0.307 & 62.45 & 19.25 & 100.94 & 0.527 & 124.05 \\
\hline $7^{2+}$ & 87.09 & 47.46 & 134.54 & 1.793 & 87.38 & 52.95 & 186.17 & 1.018 & 320.71 \\
\hline
\end{tabular}

TABLE 4. Summation of calculated ${ }^{a}$ NBO charges by ring system $^{b}$ for $2^{2+}, 5^{2+}, 6^{2+}$ and $7^{2+}$

\begin{tabular}{|c|c|c|c|c|c|c|c|}
\hline $\begin{array}{l}\Sigma \mathrm{NBO} \\
\text { charge }\end{array}$ & phenyl & ind & $\mathrm{Fl}$ & & phenyl & ind & trop \\
\hline $2 a^{2+}$ & 0.429 & 0.630 & 0.941 & $5 a^{2+}$ & 0.399 & 0.588 & 1.018 \\
\hline $2 b^{2+}$ & 0.468 & 0.601 & 0.931 & $5 b^{2+}$ & 0.434 & 0.554 & 1.012 \\
\hline $2 c^{2+}$ & 0.504 & 0.568 & 0.928 & $5 c^{2+}$ & 0.466 & 0.524 & 1.010 \\
\hline $2 d^{2+}$ & 0.541 & 0.544 & 0.921 & $5 d^{2+}$ & 0.500 & 0.495 & 1.006 \\
\hline $2 \mathrm{e}^{2+}$ & 0.651 & 0.462 & 0.887 & $5 e^{2+}$ & 0.598 & 0.404 & 0.998 \\
\hline $7^{2+}$ & & 0.999 & 1.001 & $6^{2+}$ & & 0.954 & 1.046 \\
\hline
\end{tabular}

${ }^{a}$ Charges calculated with the NPA method in Gaussian 09 with B3LYP/6-311 g(d,p) on geometries optimized with B3LYP/6-31 g(d). ${ }^{b}$ Charges from hydrogens were summed into the charges of the carbons to which they were attached.

0.651 whereas for $\mathbf{5} \mathbf{e}^{\mathbf{2}+}$ it is 0.598 , the substituents are causing a proportional response, as is shown by a linear plot of the summation of charges by substituent (see Supporting Information). One would expect that as charge is removed from the antiaromatic systems, the magnitude of the antiaromaticity would decrease; a decrease in the amount of charge would also result in a decrease of the aromaticity of the tropylium system. These changes should be apparent in changes in the NICS values for each ring system, $\Sigma$ NICS. Because the magnitude of the NICS values are related to the size of the ring, they must be divided by the square of the area, $\Sigma$ NICS/ area $^{2} .{ }^{20}$ The magnitude of delocalization of the charge in each ring system is also affected by the area. We compared $\Sigma$ NICS/area ${ }^{2}$ to $\Sigma$ NBO charges/area. The plots of $\Sigma$ NICS/ area $^{2}$ to the $\Sigma \mathrm{NBO}$ charges/area ${ }^{2}$ were very similar in terms of the quality of the correlation because there was relatively little change in the ring areas for the different systems (see Supporting Information). The plots of $\Sigma$ NICS/area ${ }^{2}$ vs $\Sigma$ NBO charges/area are given in Figure 4. Figure 4a shows the linear relationship for both indenyl systems of $\mathbf{2}^{\mathbf{2 +}}, \mathbf{5}^{\mathbf{2 +}}, \mathbf{6}^{\mathbf{2 +}}$ and $7^{2+}$; Figures $4 \mathrm{~b}$ shows the linear relationships for the fluorenyl and tropylium substituents. The positive slopes for the antiaromatic systems show that as the amount of positive charge increases, the amount of antiaromaticity increases. That is, the ability of the electron-donating substituent to stabilize the cationic system is related to a decrease in antiaromaticity.

Figure 4 suggests at least two questions. The slopes for the indenyl systems are very similar, and are around $25 \mathrm{ppm} /$ charge, but the slope of the fluorenyl system is more steep, about $60 \mathrm{ppm} / \mathrm{charge}$. The steeper slope suggests that antiaromaticity of the fluorenyl system is more sensitive to charge than the antiaromaticity of the indenyl systems. Antiaromaticity must be affected by more than simply the charge present in the system. The more obvious question is the flat slope of the tropylium system. This system, which is the only aromatic system in the species examined, shows a marked lack of responsiveness of aromaticity to changes in charge.
One possibility is that the delocalization of charge, rather than simply the amount of charge, is very important in the manifestation of aromaticity and antiaromaticity, conceivably that the greater the delocalization, the greater the aromaticity/antiaromaticity. Delocalization can be quantified by considering the lack of bond length alternation. We have previously observed with the dications and dianions of tetrabenzo[5.7]fulvalenes that aromatic systems are much less responsive to changes in the amount of bond length alternation than are antiaromatic systems. That is, when the optimized geometry for an aromatic system was modified so that the overall area was unchanged but the bond length alternation was reduced, the effect on aromaticity was very small, while the effect of similar changes for an antiaromatic system was much greater. ${ }^{7}$ Thus antiaromatic systems are much more responsive to the effectiveness of the delocalization in the system, as evaluated through bond length alternation.

Evaluation of Bond Length Alternation using the GEO Term of the Harmonic Oscillator Model of Aromaticity. Bond length alternation is the deviation of each bond length from the average bond length. This measure is considered as one term of the evaluation of structural effects on aromaticity/ antiaromaticity described as the Harmonic Oscillator Model of Aromaticity, HOMA. ${ }^{30-32}$ The HOMA approach contains two terms; the GEO term considers the effect of the degree of bond length alternation while EN term examines the deviation of the average bond length from the optimal one, $R_{\text {opt }}$, on the energy of an aromatic compound. The degree of bond length alternation is obtained by the summation of the square of the difference of each bond, $R_{\mathrm{i}}$, from the average bond length, $R_{\mathrm{av}}$, divided by the number of bonds, as shown below. For benzene both the GEO and EN terms would be 0 , thus the HOMA value would be 1 . The coefficient $\alpha$ was chosen so that the HOMA value for a localized polyene would be $0 ; n$ is the number of bonds. The larger the value of the geometric term, GEO, the greater is the degree of bond length alternation. The calculated HOMA GEO terms for $\mathbf{2}^{\mathbf{2 +}}, \mathbf{5}^{\mathbf{2 +}}, \mathbf{6}^{\mathbf{2 +}}$ and $\mathbf{7}^{\mathbf{2 +}}$ are shown in Table 5. The calculated bond lengths from which the GEO terms were determined can be found in the Supporting Information.

$$
\begin{aligned}
\mathrm{HOMA} & =1-[\mathrm{GEO}+\mathrm{EN}] \\
& =1-\left[\alpha / n \sum\left(R_{\mathrm{a} v}-R_{\mathrm{i}}\right)^{2}+\alpha\left(R_{\mathrm{opt}}-R_{\mathrm{a} v}\right)^{2}\right]
\end{aligned}
$$

(30) Kruszewski, J.; Krygowski, T. M. Tetrahedron Lett. 1972, 38393842

(31) Krygowski, T. M.; Cyranski, M. K. Tetrahedron 1999, 55, 11143 - 
a)

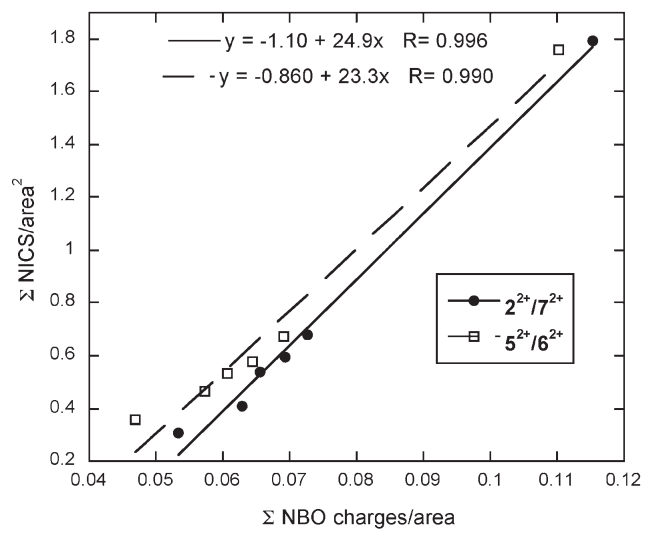

b)

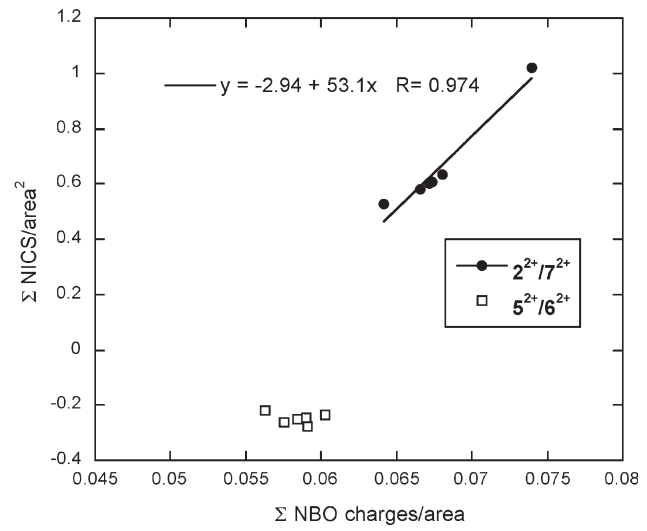

FIGURE 4. Relationship between the magnetic measure of aromaticity/antiaromaticity, corrected for the area of the ring system, $\Sigma$ NICS/sq area, with the charge of that ring system, $\Sigma$ NBO charges/area, for $\mathbf{2}^{\mathbf{2 +}}, \mathbf{5}^{\mathbf{2 +}}, \mathbf{6}^{\mathbf{2 +}}$ and $\mathbf{7}^{\mathbf{2}+}$. (a) Indenyl systems. (b) Fluorenyl $\left(\mathbf{2}^{\mathbf{2}+} / \mathbf{7}^{\mathbf{2}}\right)$ and tropylium $\left(5^{2+} / 6^{2+}\right.$ systems.

The GEO term shows the degree of bond alternation with a smaller value indicating less bond length alternation, and therefore greater delocalization. Considering first the indenyl system of $\mathbf{5}^{\mathbf{2}}$, there is an increase in bond length alternation of the entire system as the substituent $Z$ becomes more electron donating. The bond length alternation becomes greater in the 5-membered ring as the substituent becomes more electron-donating, but less in the 6-membered ring. Thus, the 6-membered ring becomes more benzene-like, more completely delocalized as the 5-membered ring becomes less completely delocalized. This suggests that the pattern of delocalization changes from that shown for $\mathbf{5}^{\mathbf{2}+}$ to that indicated as $\mathbf{5}^{\boldsymbol{*}^{\mathbf{2}+}}$. In effect, as the phenyl ring becomes more able to stabilize the carbocation, it begins to behave as an allyl cation.

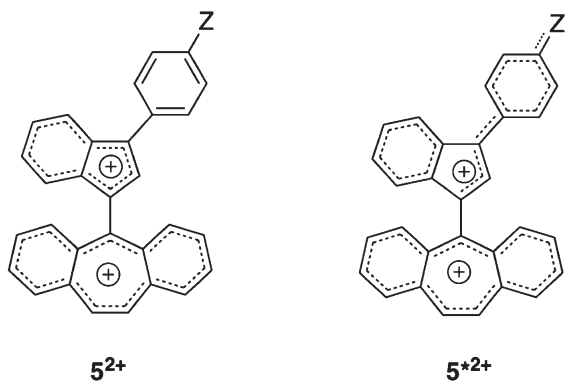

There is a similar pattern in the 6-membered ring of the indenyl system of $2^{\mathbf{2}+}$ but more variability in the 5-membered ring, and therefore in the bond length alternation of the entire indenyl system. We are inclined to attribute this variability to differences in geometry. As was demonstrated in Figure 3, the dihedral angle between the ring systems of $\mathbf{5}^{\mathbf{2}+}$ is $90^{\circ}$, which is a consequence of the greater spacial requirements of the tropylium system because of the larger bond angles in the seven-membered ring. In comparison, the dihedral angle of $\mathbf{2}^{\mathbf{2}+}$ is approximately $50^{\circ}$ (see Supporting Information). The fluorenyl ring system has smaller spacial 1419.
TABLE 5. Bond Length Alternation from Calculated Geometry of $2^{2+}$, $5^{2+}, 6^{2+}$ and $7^{2+a}$

\begin{tabular}{|c|c|c|c|c|c|c|}
\hline $5^{2+} / 6^{2+}$ & $5 a^{2+}$ & $\mathbf{5 b}^{2+}$ & $5 c^{2+}$ & $5 d^{2+}$ & $5 e^{2+}$ & $6^{2+}$ \\
\hline indenyl & 0.3577 & 0.3678 & 0.3698 & 0.3751 & 0.3893 & 0.273 \\
\hline 5-ring & 0.4220 & 0.4426 & 0.4505 & 0.4604 & 0.4970 & 0.2146 \\
\hline 6-ring & 0.1041 & 0.0968 & 0.0893 & 0.0863 & 0.0672 & 0.1997 \\
\hline tropylium & 0.2008 & 0.2010 & 0.2013 & 0.2011 & 0.2008 & 0.2087 \\
\hline 5-ring & 0.1861 & 0.1863 & 0.1866 & 0.1870 & 0.1873 & 0.2220 \\
\hline 6-ring & 0.2296 & 0.2289 & 0.2289 & 0.2274 & 0.2249 & 0.231 \\
\hline $2^{2+} / 7^{2+}$ & $2 a^{2+}$ & $2 b^{2+}$ & $2 c^{2+}$ & $2 d^{2+}$ & $2 \mathrm{e}^{2+}$ & $7^{2+}$ \\
\hline indenyl & 0.3501 & 0.355 & 0.353 & 0.35 & 0.3504 & 0.320 \\
\hline 5-ring & 0.4035 & 0.4141 & 0.4145 & 0.4184 & 0.4016 & 0.3066 \\
\hline 6-ring & 0.0504 & 0.0466 & 0.0424 & 0.0396 & 0.0311 & 0.2036 \\
\hline fluorenyl & 0.1 & 0.1809 & 0.1809 & 0.1809 & 0.1809 & 0.1821 \\
\hline 5 & 0.0958 & 0.0957 & 0.0955 & 0.0952 & 0.0943 & 0.0929 \\
\hline 6-ring & 0.0842 & 0.0829 & 0.0824 & 0.0804 & 0.0768 & 0.0971 \\
\hline
\end{tabular}

${ }^{a}$ Calculated using the geometric term from the HOMA method; $\alpha=$ 257.7; geometries optimized at the B3LYP/6-31G level.

requirements than the tropylium system because the internal bond angles of the five-membered ring of the fluorenyl system are smaller. That is, the benzene rings of the fluorenyl system are effectively tied back more than in the tropylium system. The fluorenyl and indenyl systems can move closer to planarity than was true for the tropylium and indenyl systems of $5^{2+} / \mathbf{6}^{\mathbf{2}+}$. That allows the fluorenyl system to interact with the indenyl system to a greater extent than was true for the interaction of the tropylium system with the indenyl system of $\mathbf{5}^{\mathbf{2}+} / \mathbf{6}^{\mathbf{2}+}$. A partial consequence of this might account for the greater variability of the dihedral angles for the 5-membered ring of $\mathbf{2}^{\mathbf{2 +}}$ compared to $\mathbf{5}^{\mathbf{2}}$ (see Supporting Information). Support for the change in the pattern of delocalization from $\mathbf{5}^{\mathbf{2}+}$ to $\mathbf{5}^{\mathbf{*}+}$ comes from a consideration of the NICS values. The 6-membered rings of both $\mathbf{2}^{\mathbf{2 +}}$ and $\mathbf{5}^{\mathbf{2}+}$ become more aromatic and more negative as $Z$ becomes more electron donating while the 5-membered rings become less antiaromatic.

While the indenyl systems show a change in the pattern of delocalization as a consequence of the effect of $\mathrm{Z}$, both the fluorenyl system of $\mathbf{2}^{\mathbf{2}+}$ and tropylium system of $\mathbf{5}^{\mathbf{2}+}$ show much less bond length alternation in all ring systems, therefore much greater delocalization. This is reflected in the greater antiaromaticity of both 5- and 6-membered rings of 
$\mathbf{2}^{\mathbf{2}+}$, Table 3, and greater aromaticity of both rings of $\mathbf{5}^{\mathbf{2}+}$, Table 2. The tropylium system shows much less change in NICS as a function of charge, Figure $4 b$, because it is already appreciably delocalized.

When the unsubstituted species $\mathbf{6}^{\mathbf{2 +}}$ and $\mathbf{7}^{\mathbf{2}+}$ are compared to the dications with phenyl substituents, there is greater delocalization in the indenyl systems because there is no phenyl substituent to shift the pattern of delocalization to one resembling $\mathbf{5}^{\boldsymbol{*}^{\mathbf{2}+}}$. However, both the tropylium system of $\mathbf{6}^{\mathbf{2}+}$ and the fluorenyl system of $7^{\mathbf{2}+}$ show very slightly more bond length alternation in comparison to $\mathbf{5}^{\mathbf{2 +}} / \mathbf{2}^{\mathbf{2 +}}$.

\section{Summary}

Dications $\mathbf{5 b}-\mathbf{d}^{\mathbf{2}+}$ were prepared by oxidation with $\mathrm{SbF}_{5}$ in $\mathrm{SO}_{2} \mathrm{ClF}$ and characterized by ${ }^{1} \mathrm{H}$ NMR spectroscopy. There was an excellent relationship between the experimental and calculated chemical shifts for $\mathbf{5 b}-\mathbf{d}^{\mathbf{2}+}$ as well as for previously prepared $\mathbf{2} \mathbf{a}-\mathbf{c}^{\mathbf{2}+}$, which gave validity to the NICS values calculated using the same method. The behavior of $\mathbf{2}^{\mathbf{2}+} / \mathbf{7}^{\mathbf{2}+}$, which contained indenyl and fluorenyl cationic ring systems and of $\mathbf{5}^{\mathbf{2 +}} / \mathbf{6}^{\mathbf{2}+}$, which contained indenyl and tropylium cationic ring systems was examined by a magnetic measure of aromaticity/antiaromaticity, $\Sigma$ NICS(1) $)_{z z}$. By that measure, the antiaromaticity of the indenyl ring system decreased with the introduction of a phenyl substituent in the 3-position. In addition, as substituents on that phenyl ring became more electron rich, the antiaromaticity of the indenyl ring system decreased. This suggested that the amount of charge in the system might be related to the degree of antiaromaticity. The sum of the calculated NBO charge showed a good correlation with the amount of antiaromaticity in the indenyl system. A similar examination of the fluorenyl systems of $\mathbf{2}^{\mathbf{2 +}} / \mathbf{7}^{\mathbf{2}+}$ showed a linear relationship between $\Sigma \mathrm{NICS}(1)_{z z}$ and $\Sigma \mathrm{NBO}$ charges, but in the tropylium systems of $\mathbf{5}^{\mathbf{2}+} / \mathbf{6}^{\mathbf{2}+}$, the degree of aromaticity was basically unresponsive to changes in the degree of charge. However, the responsiveness of the antiaromaticity of the fluorenyl system to changes in charge was much greater than the responsiveness of the indenyl system; the amount of charge alone was not primarily responsible for changes in antiaromaticity. To determine if the lack of bond length alternation, a measure of delocalization, might play a role, bond length alternation was calculated using the GEO term of the HOMA approach. The 5-membered ring of the indenyl system of $\mathbf{5}^{\mathbf{2}+}$ showed an increase in bond alternation while the 6-membered ring became more benzene-like and showed a change in the pattern of delocalization to $5^{\boldsymbol{*}^{\mathbf{2}+}}$. This change in the pattern of delocalization was apparent in the behavior of the 6-membered ring of $2^{2+}$ but was less clear for the 5-membered ring, presumably because of different geometric constraints in the system. Both the fluorenyl system of $\mathbf{2}^{\mathbf{2}+}$ and the tropylium system of $\mathbf{5}^{\mathbf{2}+}$ showed much greater delocalization, as shown by smaller bond length alternation. Finally, for systems without the phenyl substituent, $\mathbf{6}^{\mathbf{2}+}$ and $\mathbf{7}^{\mathbf{2}}$, the indenyl ring showed much greater delocalization than systems with the phenyl substituent. For the indenyl and fluorenyl systems, greater delocalization resulted in greater antiaromaticity, as evaluated through NICS. Aromatic systems, such as the tropylium system of $\mathbf{5}^{\mathbf{2}+} / \mathbf{6}^{\mathbf{2}+}$, were much less responsive to changes in delocalization caused by changes in the degree of charge in the system, presumably because they were already substantially delocalized.

Thus, subtle changes in electronic effects are much more visible in antiaromatic systems, as measured through bond length alternation, changes in the amount of charge, and measures of antiaromaticity. We suggest that using antiaromatic species to study delocalization is both possible to do experimentally and is a very powerful tool.

\section{Experimental Section}

The olefin precursors to $\mathbf{5} \mathbf{b}-\mathbf{e}^{\mathbf{2 +}}$ were synthesized by Peterson olefination of the appropriate substituted 3-phenyl indene ${ }^{19}$ with dibenzosuberenone, as described below for the synthesis of $\mathbf{5 b}$. 5a was prepared from 3-( $p$-bromophenyl)-1- $(1 \mathrm{H}-$ indenylidene)-5H-dibenzo[a.d]cycloheptene, which was synthesized by Peterson olefination of 1-(4-bromophenyl)-indene with dibenzosuberenone, followed by reaction with sodium trifluoroacetate in the presence of copper(I) iodide, as described below. ${ }^{1} \mathrm{H}$ and ${ }^{13} \mathrm{C}$ NMR spectra for $\mathbf{5 a}-\mathbf{e}$ can be found in the Supporting Information.

3-(Phenyl)-1-(1H-indenylidene)-5H-dibenzo $[$ a.d $]$ cycloheptene, 5b. To 1-phenyl-indene $(1.11 \mathrm{~g}, 5.77 \mathrm{mmol})$ in $40 \mathrm{~mL}$ of dry THF $-78^{\circ} \mathrm{C}$ was added $5.4 \mathrm{~mL} n$-butyllithium $(7.9 \mathrm{mmol})$, giving a dark red solution. After $1.5 \mathrm{~h}$, trimethylsilyl chloride $(1.1 \mathrm{~mL}$, $8.1 \mathrm{mmol}$ ) was added to the reaction mixture and the reaction allowed to stir for two hours, with the temperature warming to $-15^{\circ} \mathrm{C}$. An additional 1.5 equiv of $n$-butyllithium were added and the reaction mixture stirred for $2 \mathrm{~h}$. Dibenzosuberenone $1.19 \mathrm{~g}, 5.77 \mathrm{mmol}$ ) was added and stirred overnight at room temperature. The solution was quenched with water and the aqueous layer was extracted with $2 \times 50 \mathrm{~mL}$ of diethyl ether. The combined organic layers were washed with with $2 \times 25 \mathrm{~mL}$ saturated sodium bicarbonate. The solvent was removed under vacuum to yield a brown oil. Flash column chromatography with hexane was performed to yield the pure product, yellow crystals, $0.973 \mathrm{~g}, 44.2 \%$ yield. MP $172-174{ }^{\circ} \mathrm{C}$.

${ }^{1} \mathrm{H}$ NMR $\left(400 \mathrm{MHz}, \mathrm{CDCl}_{3}\right) \delta 7.53-7.62(\mathrm{~m}, 4 \mathrm{H}), 7.30-7.53$ $(\mathrm{m}, 10 \mathrm{H}), 7.17(\mathrm{tt}, J=7.52,1.18 \mathrm{~Hz}, 1 \mathrm{H}), 7.02(\mathrm{~d}, J=11.82 \mathrm{~Hz}$, $1 \mathrm{H}), 6.96(\mathrm{~d}, J=11.80 \mathrm{~Hz}, 1 \mathrm{H}), 6.90(\mathrm{t}, J=7.58 \mathrm{~Hz}, 1 \mathrm{H}), 6.60$ $(\mathrm{d}, J=1.97 \mathrm{~Hz}, 1 \mathrm{H}), 6.59(\mathrm{~d}, J=4.66 \mathrm{~Hz}, 1 \mathrm{H}) ;{ }^{13} \mathrm{C}$ NMR $\left(100 \mathrm{MHz}, \mathrm{CDCl}_{3}\right) \delta 145.0,143.7,143.2,141.5,139.3,138.5$, $137.4,136.6,135.9,134.6,134.1,131.4,131.2,128.9,128.8$, $128.8,128.7,128.6,128.3,128.2,128.1,127.9,127.4,127.4$, $126.4,125.3,124.1,120.3$. Analysis calculated for $\mathrm{C}_{30} \mathrm{H}_{20}$ : C, 94.70; H, 5.30. Found: C, 94.40; H, 5.81.

3-(p-Fluorophenyl)-1-(1H-indenylidene)-5H-dibenzo[a.d]cycloheptene, 5c. Same procedure as for $\mathbf{5 b}$, yellow crystals, 72\% yield. MP $173-174{ }^{\circ} \mathrm{C}$.

${ }^{1} \mathrm{H}$ NMR $\left(400 \mathrm{MHz}, \mathrm{CDCl}_{3}\right) \delta 7.53-7.23(\mathrm{~m}, 11 \mathrm{H}), 7.10(\mathrm{td}$, $J=7.83,1.23 \mathrm{~Hz}, 1 \mathrm{H}), 7.01(\mathrm{t}, J=8.91 \mathrm{~Hz}, 2 \mathrm{H}), 6.93(\mathrm{~d}, J=16.40$ $\mathrm{Hz}, 1 \mathrm{H}), 6.88(\mathrm{~d}, J=16.40 \mathrm{~Hz}, 1 \mathrm{H}), 6.83(\mathrm{td}, J=7.31,1.19 \mathrm{~Hz}$, $1 \mathrm{H}), 6.52(\mathrm{~d}, J=7.83 \mathrm{~Hz}, 1 \mathrm{H}), 6.47(\mathrm{~s}, 1 \mathrm{H}) ;{ }^{13} \mathrm{C}$ NMR $(100$ $\left.\mathrm{MHz}, \mathrm{CDCl}_{3}\right) \delta 164.4,161.1,144.0,143.0,139.2,138.3,137.3$, $136.5,134.6,134.1,131.9,131.3,131.2,129.6,129.5,128.8$, $128.8,128.7,128.4,128.2,128.1,127.5,126.2,125.4,124.2$, 120.1, 115.9, 115.6. Analysis calculated for $\mathrm{C}_{30} \mathrm{H}_{19} \mathrm{~F}: \mathrm{C}, 90.43$; H, 4.81; F, 4.77. Found: C, 90.06; H, 4.70.

3-(p-Methylphenyl)-1-(1H-indenylidene)-5H-dibenzo[a.d]cycloheptene, 5d. Same procedure as for $\mathbf{5 b}$, with the following exceptions: purification was achieved by column separation and recrystallization in pentane. Yellow crystals, $48.4 \%$ yield. MP $189-192{ }^{\circ} \mathrm{C}$.

${ }^{1} \mathrm{H}$ NMR $\left(400 \mathrm{MHz}, \mathrm{CDCl}_{3}\right) \delta 7.60-7.52(\mathrm{~m}, 2 \mathrm{H}) 7.52-7.31$ $(\mathrm{m}, 9 \mathrm{H}), 7.22(\mathrm{t}, J=8.47 \mathrm{~Hz}, 1 \mathrm{H}), 7.20(\mathrm{t}, J=6.85 \mathrm{~Hz}, 1 \mathrm{H}), 7.17$ $(\mathrm{t}, J=7.48 \mathrm{~Hz}, 1 \mathrm{H}), 7.01(\mathrm{~d}, J=11.8 \mathrm{~Hz}, 1 \mathrm{H}), 6.96(\mathrm{~d}, J=11.8 \mathrm{~Hz}$, $1 \mathrm{H}), 6.89(\mathrm{t}, J=7.62 \mathrm{~Hz}, 1 \mathrm{H}), 6.58(\mathrm{~d}, J=8.02 \mathrm{~Hz}, 1 \mathrm{H}), 6.57(\mathrm{~s}, 2$, 
$1 \mathrm{H}), 2.38(\mathrm{~s}, 3 \mathrm{H}) ;{ }^{13} \mathrm{C} \mathrm{NMR}\left(100 \mathrm{MHz}, \mathrm{CDCl}_{3}\right) \boldsymbol{\delta} 145.0,143.1$, $139.3,138.5,138.0,137.5,136.6,134.6,134.1,133.0,131.3$, $131.2,129.4,128.9,128.8,128.8,128.7,128.3,128.1,128.0,127.8$ $127.4,127.4,125.9,125.2,124.1,120.3,21.4$. Analysis calculated for $\mathrm{C}_{29} \mathrm{H}_{20}$ : C, 94.38; H, 5.47. Found: $\mathrm{C}, 94.01 ; \mathrm{H}, 5.60$.

3-(p-Methoxyphenyl)-1-(1H-indenylidene)-5H-dibenzo[a.d]cycloheptene, 5e. Same procedure as for $\mathbf{5 b}$ yellow crystals, $37 \%$ yield. MP $204-205.5^{\circ} \mathrm{C}$.

${ }^{1} \mathrm{H}$ NMR $\left(400 \mathrm{MHz}, \mathrm{CDCl}_{3}\right) \delta 7.60-7.30(\mathrm{~m}, 9 \mathrm{H}), 7.42-7.31$ $(\mathrm{m}, 2 \mathrm{H}), 7.17(\mathrm{tt}, J=7.52,1.26 \mathrm{~Hz}, 1 \mathrm{H}), 7.02(\mathrm{~d}, J=11.8 \mathrm{~Hz}, 1 \mathrm{H})$, $6.96(\mathrm{~d}, J=11.8 \mathrm{~Hz}, 1 \mathrm{H}), 6.94(\mathrm{~d}, J=7.56 \mathrm{~Hz}, 2 \mathrm{H}), 6.89(\mathrm{t}, J=$ $7.62 \mathrm{~Hz}, 1 \mathrm{H}) ;{ }^{13} \mathrm{C} \mathrm{NMR}\left(400 \mathrm{MHz}, \mathrm{CDCl}_{3}\right) \boldsymbol{\delta} 159.6,144.6,143.5$ $143.0,139.4,138.5,137.5,136.6,134.6,134.2,131.3,131.2$ $129.1,128.9,128.8,128.8,128.7,128.3,128.1,127.4,126.1$, $125.3,125.2,123.9,120.3,114.4,55.4$. Analysis calculated for $\mathrm{C}_{31} \mathrm{H}_{22} \mathrm{O}$ : C, 90.70; H, 5.40; O, 3.90. Found: C, 90.34; H, 5.38.

3-(p-Trifluoromethylphenyl)-1-(1H-indenylidene)-5H-dibenzo[a.d]cycloheptene, 5a. A solution of 3-(p-bromophenyl)-1- $(1 \mathrm{H}-$ indenylidene)-5H-dibenzo[a.d]cycloheptene $(0.4594 \mathrm{~g}, 1.000 \mathrm{mmol})$ in $40 \mathrm{~mL}$ of dry $N$-methyl-2-pyrrolidone was purged with argon for $30 \mathrm{~min}$. Copper(I) iodide $(0.762 \mathrm{~g}, 4.000 \mathrm{mmol})$, which had been purified by Soxhet extraction with $\mathrm{CH}_{2} \mathrm{Cl}_{2}$, and $\mathrm{NaO}_{2-}$ $\mathrm{CCF}_{3}(1.08 \mathrm{~g}, 8.00 \mathrm{mmol})$ were added and the solution refluxed under Ar overnight. After cooling to room temperature, $250 \mathrm{~mL}$ ether was added and the reaction mixture filtered through Celite. The solution was extracted with twice with water and twice with brine. The solution was dried, solvent removed under vacuum, and the product isolated by chromatography on silica with gradient eleution of hexanes to $5 \% \mathrm{CH}_{2} \mathrm{Cl}_{2} /$ hexanes to give yellow crystals. Yield: $0.250 \mathrm{~g}, 55.8 \%$. MP $187-191^{\circ} \mathrm{C}$.

$\mathrm{H}^{1} \mathrm{NMR}\left(\mathrm{CDCl}_{3}\right.$, TMS internal standard, $\left.300 \mathrm{MHz}\right)$ : $\delta 7.67-7.64(4 \mathrm{H}, \mathrm{m}), \delta 7.61-7.39(9 \mathrm{H}, \mathrm{m}) 7.19(\mathrm{t}, J=7.62 \mathrm{~Hz}$, $1 \mathrm{H}), 7.02(2 \mathrm{H}, \mathrm{d}, J=4.69 \mathrm{~Hz}) 6.95(1 \mathrm{H}, \mathrm{t}, J=7.62 \mathrm{~Hz}), 6.66(1 \mathrm{H}$, s), $6.66(1 \mathrm{H}, \mathrm{d}, J=8.20 \mathrm{~Hz}) .{ }^{13} \mathrm{C}$ NMR $\left(100 \mathrm{MHz}, \mathrm{CDCl}_{3}\right)$ $\delta 145.1,142.5,139.0,138.2,137.1,136.3,134.5,134.0,131.3$, $131.2,128.9,128.8,128.7,128.6,128.4,128.3,128.1,127.6$, $127.6,125.7,125.7,125.6,124.3,120.0$. Analysis calculated for $\mathrm{C}_{33} \mathrm{H}_{19} \mathrm{~F}_{3}$ : C, 83.02; H, 4.27; F, 12.71. Found: C, 82.90; H, 4.28.

Preparation of Dications by Chemical Oxidation. $\mathrm{SbF}_{5}(\sim 0.7$ $\mathrm{mL}, \sim 9 \mathrm{mmol}$ ) was added to a graduated centrifuge tube in a drybox and the tube was capped with a septum and placed in an ice bath. $\mathrm{SO}_{2} \mathrm{ClF}^{33}(1.3 \mathrm{~mL})$ at $-78^{\circ} \mathrm{C}$ was transferred by cannula into the centrifuge tube. The contents were mixed on

(33) Reddy, V. P.; Bellew, D. R.; Prakash, G. K. S. J. Fluorine Chem 1992, 56, 195-197. a vortex stirrer until homogeneous, and the solution was cooled to $-78^{\circ} \mathrm{C}$. The neutral precursor $(\sim 3 \mathrm{mmol})$ was added in small portions, followed by vortex mixing and cooling to $-78{ }^{\circ} \mathrm{C}$. Samples for NMR analysis were kept at $-78^{\circ} \mathrm{C}$ until needed and transferred by chilled pipet into a chilled NMR tube. A capillary tube with acetone- $d_{6}$ was then inserted into the NMR tube to serve as an external standard and deuterium lock.

\section{Computational Methods}

Geometries were optimized at B3LYP/6-31G(d) density functional theory levels with the Gaussian 98 and 03 program packages, (see Supporting Information). The chemical shifts were calculated at $\mathrm{B} 3 \mathrm{LYP} / 6-311+\mathrm{G}(\mathrm{d}, \mathrm{p})$ using the GIAO approach with the Gaussian 98 or 03 program packages on the optimized geometries. The best agreement between experimental and calculated shifts was found when the effect of solvent was included in the calcuation through the polarization continuum method, PCM. The nucleus-independent chemical shifts $\left(\operatorname{NICS}(1)_{z z}\right)^{23,24}$ were obtained from the chemical shift tensor perpendicular to the ring for a dummy atom placed $1 \AA$ above the center of each ring. Natural bond order, $\mathrm{NBO}$, charges were calculated using the natural population analysis ${ }^{27}$ in Gaussian 09 with basis set $\mathrm{B} 3 \mathrm{LYP} / 6-311+\mathrm{g}(\mathrm{d}, \mathrm{p})$ on geometries optimized as described above. Areas were calculated from the Cartesian coordinates for each ring system oriented in the xy plane. See Supporting Information for specific details of the calculation.

Acknowledgment. We thank the Welch Foundation, grant W-794, and the National Science Foundation, grant CHE0242227 and CHE-0948445 for support of this work.

Supporting Information Available: Spectra of $\mathbf{5 a}, \mathbf{c}, \mathrm{e}^{\mathbf{2 +}}$, plots of experimental vs calculated shifts for $\mathbf{2 a}-\mathbf{c}^{2+}$ and for $\mathbf{2 a}-\mathbf{c}^{\mathbf{2 +}} /$ $\mathbf{5 b}, \mathbf{d}^{\mathbf{2}}$, table of the calculated NBO charges for each atom of $\mathbf{2}^{\mathbf{2 +}}, \mathbf{5}^{\mathbf{2}+}, \mathbf{6}^{\mathbf{2 +}}$ and $\mathbf{7}^{\mathbf{2 +}}$, plot of summation of charges by substituent, plots of $\Sigma$ NICS $(1)_{z z} / \operatorname{area}^{2}$ vs NBO charge/area, by indenyl systems and by fluorenyl/tropylium systems, calculated bond lengths for $\mathbf{2}^{\mathbf{2}}, \mathbf{5}^{\mathbf{2 +}}, \mathbf{6}^{\mathbf{2}}$ and $\mathbf{7}^{\mathbf{2 +}}$, relationship between $\sum$ NICS $(1)_{z z} /$ area $^{2}$ and the GEO term for $\mathbf{2}^{\mathbf{2}+}$ and $\mathbf{5}^{\mathbf{2}+},{ }^{1} \mathrm{H}$ and ${ }^{13} \mathrm{C}$ NMR spectra for $\mathbf{5 a}-\mathbf{e}$, calculated ring areas including details of their calculation, complete reference for Gaussian 98, Gaussian 03, and Gaussian 09, Cartesian coordinates, total energies for $\mathbf{5 a}-\mathbf{e}^{\mathbf{2 +}}, \mathbf{6}^{\mathbf{2 +}}$ and $\mathbf{7}^{\mathbf{2}+}$. This material is available free of charge via the Internet at http://pubs.acs.org. 